Results Participants were allocated to vitamin $\mathrm{D}_{3}$ vs. placebo in equal numbers; $82 \%$ were vitamin $\mathrm{D}$ insufficient at baseline. Vitamin $\mathrm{D}_{3}$ supplementation did not influence time to first severe exacerbation (aHR 1.02, 95\% CI 0.69-1.53, P = 0.91) or time to first URI (aHR $0.87,95 \%$ CI $0.64-1.16, \mathrm{P}=0.34$ ). The influence of vitamin $\mathrm{D}_{3}$ on co-primary outcomes was not modified by baseline vitamin $\mathrm{D}$ status or genotype. Of 16 prespecified secondary outcomes, only one showed a difference between arms: vitamin $\mathrm{D}$ supplementation induced a modest improvement in respiratory quality of life as evidenced by a reduction in mean total score for the St George's Respiratory Questionnaire at 2 months ( -3.9 points, $\mathrm{p}=0.005), 6$ months $(-3.7$ points, $\mathrm{p}=0.038)$ and 12 months $(-3.3$ points, $\mathrm{p}=0.060)$.

Conclusions Vitamin $\mathrm{D}_{3}$ supplementation did not influence time to exacerbation or URI in a population of adults with ICStreated asthma with a high prevalence of baseline vitamin D insufficiency.

\section{S96 BRONCHIAL THERMOPLASTY REDUCES PERIPHERAL BLOOD EOSINOPHILS IN SEVERE ASTHMA DEMONSTRATING SYSTEMIC EFFECTS OF A LOCALISED THERAPY}

DM Ryan, U Holmes, G McCumesky, RD Daly, K Hince, G Tavernier, S Fowler, RM Niven. University Hospital South Manchester, Manchester, UK

\subsection{6/thoraxjnl-2014-206260.102}

Introduction Severe Asthma, characterised by persistent symptoms despite maximal medical therapy, represents $5 \%$ of asthma cases. Bronchial Thermoplasty (BT) is a novel therapy, NICE approved for Severe Asthma patients uncontrolled despite step 4/5 of British Guideline on Asthma Management. BT delivers radiofrequency thermal energy to airways distal to the mainstem bronchi, permanently reducing airway smooth muscle mass. It is unknown whether treatment of smooth muscle hypertrophy impacts persistently upon systemic signs of allergic inflammation. Peripheral blood eosinophils (PBEs) are a marker of allergic inflammation in asthma. We asked: does BT modify signs of allergic inflammation as measured by PBEs and if so, does this effect persist over time?

Method A retrospective review of 15 consecutive Severe Asthma cases treated with BT was performed. Serial PBEs measured

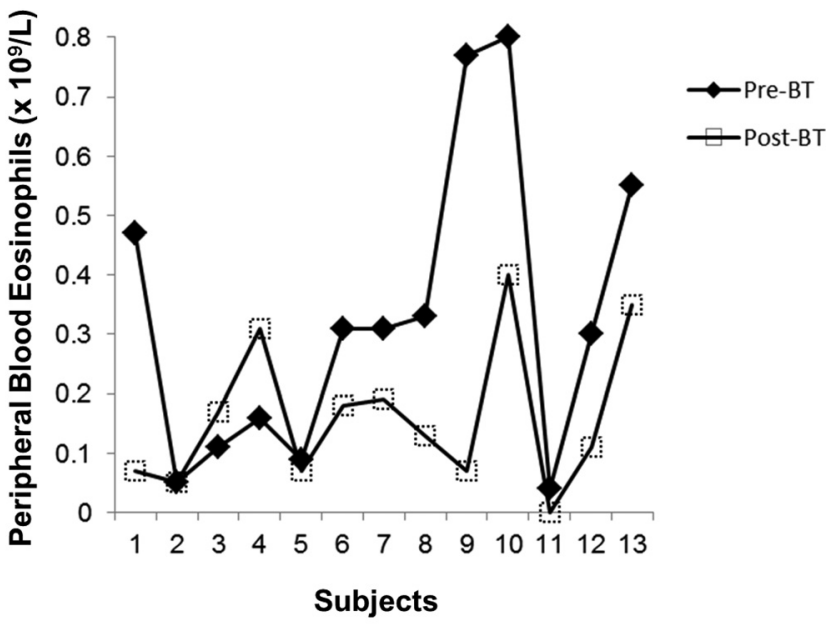

Abstract S96 Figure 1 before and up to 1 year after BT were compared. Blood eosinophil levels taken peri-procedure were excluded from analysis due to standard protocol concomitant steroid therapy. For time to first detectable high PBE all available post-BT PBE levels were assessed.

Results 13 patients had PBE data before and after BT, with an average of 9 and 12 serial PBE levels pre and post-BT respectively. Mean PBE 1 year pre-BT was $0.33 \times 10^{9} / \mathrm{L}$ falling to a mean of $0.16 \times 10^{9} / \mathrm{L} 1$ year post-BT $(\mathrm{p}<0.05)$ (see Figure). 9 of 13 patients had a fall in mean PBE, in 2 of 13 levels rouse and 1 of 13 mean PBEs were unchanged post-BT. In 6 patients who converted from normal to high PBE post-BT, average time to first high $\mathrm{PBE}\left(>0.4 \times 10^{9} / \mathrm{L}\right)$ was 7 months (range 1-13 months). In 5 patients (38\%) PBE remained within normal range persistently post BT.

Conclusion Severe Asthma patients undergoing BT had a significant reduction in average peripheral blood eosinophil levels from baseline. In over $1 / 3$ of cases this effect was persistent 1 year post procedure. These findings support the concept that BT not only reduces asthma-associated smooth muscle hypertrophy but impacts upon systemic markers of allergic inflammation.

\section{Mechanistic insights in acute lung injury}

\section{S97 LONG TERM SURVIVAL IN PATIENTS WHO UNDERGO OESOPHAGECTOMY IS LOWER IN PATIENTS WHO DEVELOP POST-OPERATIVE ACUTE RESPIRATORY DISTRESS SYNDROME}

${ }^{1}$ RCA Dancer, ${ }^{1} \mathrm{D}$ Parekh, ${ }^{2} \mathrm{GD}$ Perkins, ${ }^{1} \mathrm{DR}$ Thickett. ${ }^{1}$ University of Birmingham, Birmingham, UK; ${ }^{2}$ University of Warwick, Coventry, UK

\subsection{6/thoraxjn|-2014-206260.103}

Oesophagectomy is a complicated procedure with high risk of complications in the immediate post-operative period. We have previously shown that patients undergoing oesophagectomy have $\sim 25 \%$ risk of Acute Respiratory Distress Syndrome (ARDS) post op. Post-operative complications have been shown to decrease long term post-operative survival following major surgery. ${ }^{1}$ We hypothesised that long term survival would be reduced in patients who develop ARDS post oesophagectomy.

We analysed data from 55 patients recruited to the translational sub-study of the BALTI prevention trial. 26 of the 55 patients $(47 \%)$ died within 2 years of their operation.

Patients who died within two years of their oesophagectomy were more likely to have required ventilation for ARDS during their hospital admission. In addition, patients who survived less than two years were more likely to have developed a surgical complication (e.g. anastomotic leak, wound infection, chyle leak) post-op. There was no difference in age, lung function, BMI or cancer staging. Patients who did not survive more than 2 years post-op were more likely to be smoking at the time of the operation, but there was no difference in pack year smoking history between the two groups.

Perioperative markers of alveolar epithelial damage (PICCO EVLWI and PVPI), and the severity of both local (BAL CRP) and systemic inflammation (IL-17, ICAM-1, and TNFR-1/2) were associated with outcome.

In conclusion, complications during recovery from oesophagectomy have an adverse effect on the chances of long term survival. Development of strategies to reduce post-operative morbidity may improve long term outcomes. 
Abstract S97 Table 1 Patients who die within two years of oesophagectomy are more likely to have been smoking at the time of their operation, or to have developed ARDS or a surgical complication in the immediate post-op period

\begin{tabular}{llll}
\hline & $\begin{array}{l}\text { Died within } 2 \text { years } \\
(\mathrm{n}=26)\end{array}$ & $\begin{array}{l}\text { Survived 2 years } \\
(\mathrm{n}=29)\end{array}$ & \begin{tabular}{l} 
P-value \\
\hline ARDS $-\mathrm{n}(\%)$
\end{tabular} \\
Surgical Complication $-\mathrm{n}(\%)$ & $11(42)$ & $3(10)$ & 0.007 \\
Current Smoker $-\mathrm{n}(\%)$ & $13(50)$ & $6(21)$ & 0.022 \\
Median Pack Years & $10(43)$ & $4(15)$ & 0.024 \\
\hline
\end{tabular}

\section{REFERENCES}

1 Khuri et al. Determinants of Long-Term Survival After Major Surgery and the Adverse Effect of Postoperative Complications. Ann Surg. 2005 Sep;242(3):32641

\section{S98 A NOVEL HUMAN MODEL TO STUDY ALVEOLAR INJURY AND REPAIR}

J Alçada, JP Ng-Blichfeldt, AG Proudfoot, MJD Griffiths, CH Dean, M Hind. Leucocyte Biology, National Heart and Lung Institute, Imperial College London, London, UK

\subsection{6/thoraxjnl-2014-206260.104}

Introduction The development of regenerative therapies holds promise for the future treatment of parenchymal lung diseases. However, encouraging preclinical data from animal models have translated poorly in clinical trials. The cellular and molecular response to lung injury is difficult to study in man. To address this fundamental question, we have developed a novel in vitro human model. Precision cut lung slice (PCLS) culture is a wellestablished tool in airway biology and pharmacology. Here, we demonstrate lung parenchyma can be maintained and manipulated in vitro generating a tractable model, which allows study of lung injury and repair in man.

Methods PCLS $(500 \mu \mathrm{m})$ were generated from agarose-inflated lung lobes from human lungs maintained ex-vivo by perfusion and ventilation (EVLP). The slices were cultured in serum-free medium in a rotating incubator $\left(37^{\circ} \mathrm{C}, 5 \% \mathrm{CO}_{2}\right)$ and analysed at days 1, 3 and 7. Cell specific immunofluorescence markers were used to identify smooth muscle, type I and type II alveolar epithelial cells (AT1, AT2), vascular endothelial cells and proliferating cells (using $\alpha \mathrm{SMA}$, Aquaporin5, ProSPC, PECAM1 and Phospho-histone $\mathrm{H} 3$ respectively). Slice viability was confirmed using MitoTracker, LDH and Live/Dead assays.

Results All of the expected cell types were identified in PCLS by immunofluorescence demonstrating that human PCLS maintained cellular differentiation in culture. Pro-SPC was predominant in the alveolar wall cells, particularly in the alveolar septal junctions, corresponding to known location of AT2 cells; AQ5 was distributed in thin bands lining the alveolar walls suggestive of the apical membrane of AT1 cells; $\alpha \mathrm{SMA}$ was positive around airways, the known location of smooth muscle cells (SMCs); PECAM-1 was positive within alveolar walls corresponding to microvascular capillaries within alveolar septae. There was no significant cell proliferation during culture under basal conditions. Finally, cell viability studies demonstrated that PCLS can be maintained for up to 1 week in serum-free culture.

Conclusion PCLS of human lung parenchyma remain differentiated and viable for up to 7 days in serum-free culture. In future, human PCLS derived from normal and injured regions of lung from the EVLP model may provide a novel means of studying alveolar repair in human lung in vitro. \begin{tabular}{|l|l}
\hline S99 & EFFECTS OF DIFFERENTIAL TNF RECEPTOR SIGNALLING
\end{tabular} IN MODULATING NEUTROPHIL-ENDOTHELIAL INTERACTIONS IN THE PULMONARY MICROVASCULATURE

${ }^{1}$ AG Proudfoot, ${ }^{2}$ J Juss, ${ }^{2}$ S Appleby, ${ }^{3}$ P Morley, ${ }^{3}$ J Cordy, ${ }^{3}$ A Bayliffe, ${ }^{1} M$ Hind, ${ }^{2}$ ER Chilvers, ${ }^{1} \mathrm{M}$ Griffiths, ${ }^{2} \mathrm{C}$ Summers. ${ }^{1}$ Imperial College, London, UK; ${ }^{2}$ University of Cambridge, Cambridge, UK; ${ }^{3}$ GlaxoSmithKline, Stevenage, UK

\subsection{6/thoraxjnl-2014-206260.105}

Neutrophil recruitment into the bronchoalveolar space is central to the pathogenesis of acute respiratory distress syndrome injury (ARDS), and occurs via interaction with the lung microvascular endothelium. Tumour Necrosis Factor (TNF) is a key mediator in these processes, activating endothelial cells and inducing changes in microvascular permeability, as well as priming neutrophils (a pre-requisite for neutrophil-mediated tissue damage) and modulating neutrophil lifespan. TNF signals through two cell surface receptors, TNFR1 and TNFR2 initiating distinct signalling pathways and cellular responses. In a human in vivo model of ARDS, selective TNFR1 antagonism attenuated pulmonary inflammation (O'Kane et al, Thorax 2013; 63:A50). Using TNF receptor specific muteins and a novel highly selective TNFR1 antagonist, we investigated the role of differential TNFR signalling on neutrophil-pulmonary microvascular endothelial cell interactions.

TNF-induced alterations in the expression of the neutrophil cell surface molecules CD11b, CD62L, TNFR1 and TNFR2 were all modulated via TNFR1. TNFR1 was also the dominant receptor mediating reactive oxygen species generation by TNFprimed, fMLP-stimulated neutrophils. We further examined the role of TNF receptors in modulating neutrophil apoptosis; whilst engagement of both TNFR1 and 2 was required to induce early neutrophil apoptosis, TNFR1 antagonism reversed TNF-induced late survival to constitutive levels of apoptosis. TNFR1 antagonsim of human pulmonary microvascular endothelial monolayers significantly reduced TNF-induced production of IL-1beta, IL-6 and IL-8 $(\mathrm{p}<0.05)$, endothelial permeability and the release of the endothelial injury markers sICAM-1, sVCAM-1 and sE-selectin $(\mathrm{p}$

Collectively, these results suggest that TNFR1 regulates multiple components of neutrophil-endothelial interactions. Selective TNFR1 antagonism may offer a novel therapeutic approach in ARDS; phase II clinical trials of this therapy are scheduled.

\section{S100 PROTEINASE-ACTIVATED RECEPTOR 1 SIGNALLING CONTRIBUTES TO NEUTROPHILIC INFLAMMATION AND ALVEOLAR BARRIER DISRUPTION IN STREPTOCOCCUS PNEUMONIAE PNEUMONIA}

${ }^{1} \mathrm{RJ} J$ José, ${ }^{1} \mathrm{AE}$ Williams, ${ }^{1} \mathrm{MG}$ Sulikowski, ${ }^{2} \mathrm{DA}$ Brealey, ${ }^{1} \mathrm{JS}$ Brown, ${ }^{1} \mathrm{RC}$ Chambers. ${ }^{1}$ Centre for Inflammation and Tissue Repair, Division of Medicine, University College London, London, UK; ${ }^{2}$ Critical Care, University College London Hospital, London, UK

\subsection{6/thoraxjnl-2014-206260.106}

Introduction Streptococcus pneumoniae is the most common cause of community-acquired pneumonia (CAP) and is associated with excessive neutrophilic inflammation. The high-affinity thrombin receptor, proteinase-activated receptor (PAR)-1, has been implicated in mediating the interplay between coagulation and inflammation. However, its role during S. pneumoniaeinduced neutrophilic inflammation and the mechanisms for neutrophil recruitment in this context are poorly understood. 